\title{
Correlation and Regression Analysis of 2N Scattering Observables
}

Received: 10 May 2021 / Accepted: 25 August 2021 / Published online: 8 September 2021

(C) The Author(s) 2021

\begin{abstract}
This contribution provides information about correlations among the neutron-proton $(n p)$ elastic scattering observables. The most basic prerequisite for this study is existence of models of the nucleon-nucleon $(\mathrm{NN})$ force, for which a covariance matrix of potential parameters is available. Using the covariance matrices for the chiral SMS NN potential from the Bochum-Bonn group and the one for the OPE-Gaussian potential proposed by the Granada group, we performed a systematic analysis of the correlation coefficients between the differential cross section and the depolarization $R$, as well as between $R$ and the polarization $P$.
\end{abstract}

\section{Introduction}

One of the main goals of theoretical low-energy nuclear physics is to establish the structure of the nuclear Hamiltonian. Currently, effective and phenomenological models of nuclear interactions are still of great importance. The examples of such models are: the semiphenomenological One-Pion-Exchange Gaussian (OPE-Gaussian) potential, proposed by the Granada group [1] and the chiral interaction derived up to the fifth-order $\mathrm{N}^{4} \mathrm{LO}$ of the chiral expansion using the semilocal regularization in momentum space (SMS) by the Bochum-Bonn group [2]. This choice is dictated by the availability of the covariance matrix for the free parameters of these forces. The knowledge of the covariance matrix of the potential parameters helps to investigate correlations among various two- or three-nucleon $(2 \mathrm{~N} / 3 \mathrm{~N})$ observables as well as between observables and specific potential parameters. The information about correlations among such observables is particularly interesting in the context of determining free strength parameters present in the $3 \mathrm{~N}$ interaction. However, the study of the correlations in the $2 \mathrm{~N}$ system is also interesting and can impact future procedures used to fix free parameters of the NN force. In this paper, we continue our previous studies [3], [4], [5] observables in the $2 \mathrm{~N}$ system.

In the past a study of the correlation, at a statistically significant level, was not possible due to the lack of a sufficient number of NN potentials and data points. Recently, this situation has changed. Using the OPEGaussian or the chiral SMS forces allows us to prepare many sets of the potential parameters. This can be done in the following way. Having at our disposal the covariance matrix for the potential parameters, as well as the central values of the parameters, we sample 50 sets of the potential parameters from the multivariate normal distribution. This number of sets is sufficient to analyze the theoretical uncertainties and to draw sound conclusions, as was shown in Refs. [6], [7]. In addition, 50 sets of the potential parameters are sufficient to estimate correlation coefficients among scattering observable and qualitatively interpret their values. For both $\mathrm{NN}$ forces and each set of the potential parameters we computed the deuteron wave function by solving the Schrödinger equation and the $t$-matrix elements from the Lippmann-Schwinger equation. For more details, we refer to Ref. [8].

Y. Volkotrub (凶) J J. Golak · R. Skibiński · K. Topolnicki · H. Witała

Jagiellonian University: Uniwersytet Jagiellonski w Krakowie, Kraków, Poland

E-mail: yuvolkotrub@gmail.com 


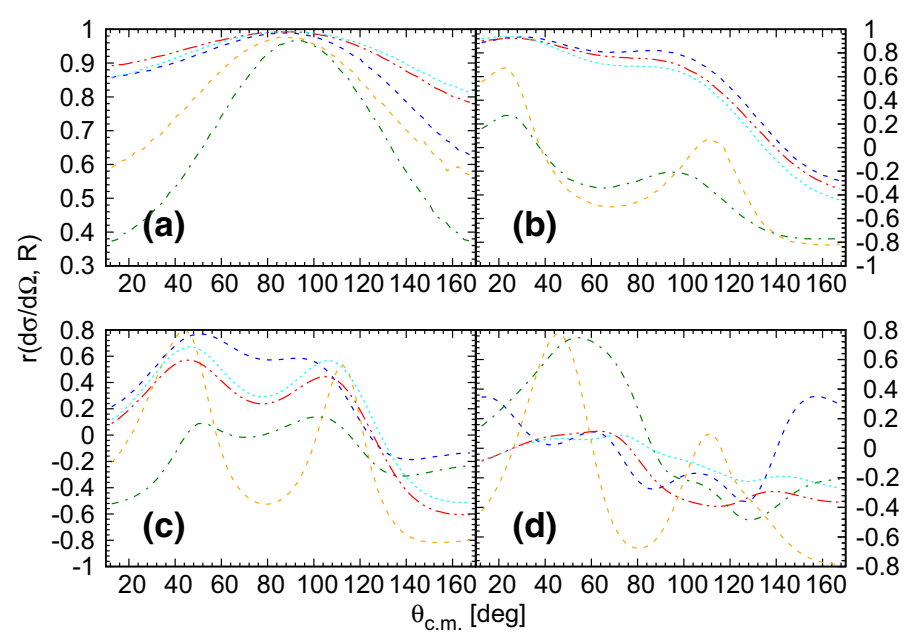

Fig. 1 The angular dependence of correlation coefficients $r$ between $d \sigma / d \Omega$ and $R$ in $n p$ scattering at the incoming neutron laboratory energy a $E_{\text {lab }}=10 \mathrm{MeV}$, b $E_{\text {lab }}=30 \mathrm{MeV}$, c $E_{\text {lab }}=65 \mathrm{MeV}$, and $\mathbf{d} E_{\mathrm{lab}}=135 \mathrm{MeV}$. The dashed orange, dash-dotted green, red double-dot-dashed, cyan dotted lines represent predictions of the chiral $\mathrm{N}^{2} \mathrm{LO}, \mathrm{N}^{3} \mathrm{LO}, \mathrm{N}^{4} \mathrm{LO}$, and $\mathrm{N}^{4} \mathrm{LO}^{+}$ SMS forces, respectively, and the blue dashed line shows predictions based on the OPE-Gaussian potential

\section{Results}

Elastic scattering of particles with spin 1/2 offers much more diverse measurements than only of the unpolarized cross section. Various spin observables, in particular the depolarization $R$ and the polarization $P$, yield important information about the NN interaction. For each version of the chiral SMS NN potentials at $\mathrm{N}^{2} \mathrm{LO}$, $\mathrm{N}^{3} \mathrm{LO}, \mathrm{N}^{4} \mathrm{LO}$ and $\mathrm{N}^{4} \mathrm{LO}^{+}$with the cutoff parameter $\Lambda=450 \mathrm{MeV}$, and the OPE-Gaussian potential, we computed mentioned observables as well as their correlation coefficients at four incident neutron energies $E_{\mathrm{lab}}=10,30,65$, and $135 \mathrm{MeV}$ in the range of the scattering angle $\theta_{c . m} . \in\left[12.5^{\circ}, 167.5^{\circ}\right]$.

Figures 1 and 2 shows examples of the angular dependence of correlation coefficient for $(d \sigma / d \Omega, R)$ and $(P, R)$ pairs. As seen from Fig. 1, the $n p$ differential cross section $d \sigma / d \Omega$ is, in general, strongly correlated with $R$ over a wide range of scattering angle at the incoming neutron laboratory energies $E_{\text {lab }}=10 \mathrm{MeV}$ and 30 $\mathrm{MeV}$. The magnitude of the correlation coefficient between the observables exceeds 0.8 . For the higher energies $\left(E_{\text {lab }}=65 \mathrm{MeV}\right.$ and $135 \mathrm{MeV}$ ) the correlation decreases, but still at some regions correlation is moderate. This is especially clearly seen for the chiral $\mathrm{N}^{4} \mathrm{LO}$ and $\mathrm{N}^{4} \mathrm{LO}^{+} \mathrm{SMS}$, and the OPE-Gaussian predictions.

In the case of $P$ and $R$, see Fig. 2, one gets a weak correlation for each of $\mathrm{N}^{4} \mathrm{LO}, \mathrm{N}^{4} \mathrm{LO}^{+} \mathrm{SMS}$ and the OPEGaussian potentials for most of scattering angles and at all energies. The exceptional, maxima of correlation coefficients $r$ are observed with $r \approx 0.65$ for $\mathrm{N}^{4} \mathrm{LO}$ force $\left(r \approx 0.6\right.$ for $\left.\mathrm{N}^{4} \mathrm{LO}^{+}\right)$in $\theta_{\text {c.m. }} \in\left(60^{\circ}, 80^{\circ}\right)$ for $E_{\text {lab }}=65 \mathrm{MeV}$. At $E_{\text {lab }}=135 \mathrm{MeV}$ the correlation coefficient can reach 0.8 at very backward scattering angles for the chiral force at $\mathrm{N}^{4} \mathrm{LO}^{+}$order.

In Figs. 3 and 4 we demonstrate results on dependences between $2 \mathrm{~N}$ scattering observables based on the two models (the OPE-Gaussian and the chiral $\mathrm{N}^{4} \mathrm{LO}^{+}$SMS potentials) using 50 sets of potential parameters by plotting the scatter plots for pairs of observables at a given scattering angle. The top panel in Fig. 3 visualizes a strong positive correlation between $d \sigma / d \Omega$ and $R$ at $E_{\text {lab }}=10 \mathrm{MeV}$ and at three scattering angles $\theta_{\text {c.m. }}=$ $30^{\circ}, 90^{\circ}, 150^{\circ}$ with the corresponding magnitudes of the correlation coefficients $r\left(\theta_{c . m} .=30^{\circ}\right)=0.89$, $r\left(\theta_{c . m .}=90^{\circ}\right)=0.99, r\left(\theta_{c . m .}=150^{\circ}\right)=0.86$ for the chiral $\mathrm{N}^{4} \mathrm{LO}^{+}$SMS force $\left(r\left(\theta_{c . m .}=30^{\circ}\right)=0.88\right.$, $r\left(\theta_{c . m .}=90^{\circ}\right)=0.99, r\left(\theta_{c . m .}=150^{\circ}\right)=0.72$ for the OPE-Gaussian potential). For $E_{\text {lab }}=135 \mathrm{MeV}$ and the three scattering angles, the scatter plots in the bottom row of Fig. 3, indicate a weak correlation between the pair $(d \sigma / d \Omega, R)$ for both potentials.

The relation between $P$ and $R$ constitutes a case of uncorrelated observables (Fig. 4). Here, at $E_{\text {lab }}=$ $10 \mathrm{MeV}$ the correlation coefficients are $r\left(\theta_{c . m .}=30^{\circ}\right)=-0.4, r\left(\theta_{\text {c.m. }}=90^{\circ}\right)=-0.19, r\left(\theta_{\text {c.m. }}=150^{\circ}\right)=$ 0.03 for the chiral $\mathrm{N}^{4} \mathrm{LO}^{+} \mathrm{SMS}$ force $\left(r\left(\theta_{c . m .}=30^{\circ}\right)=-0.02, r\left(\theta_{c . m .}=90^{\circ}\right)=0.11, r\left(\theta_{c . m .}=150^{\circ}\right)=\right.$ 0.19 for the OPE-Gaussian potential). But at $E_{\text {lab }}=135 \mathrm{MeV}$ we observe a significant increase of the magnitude of $r$, i.e. $r\left(\theta_{c . m .}=30^{\circ}\right)=0.50, r\left(\theta_{c . m .}=90^{\circ}\right)=0.56, r\left(\theta_{c . m .}=150^{\circ}\right)=-0.86$ for the chiral $\mathrm{N}^{4} \mathrm{LO}^{+}$SMS force $\left(r\left(\theta_{c . m .}=30^{\circ}\right)=0.25, r\left(\theta_{c . m .}=90^{\circ}\right)=0.54, r\left(\theta_{c . m .}=150^{\circ}\right)=-0.83\right.$ for the 


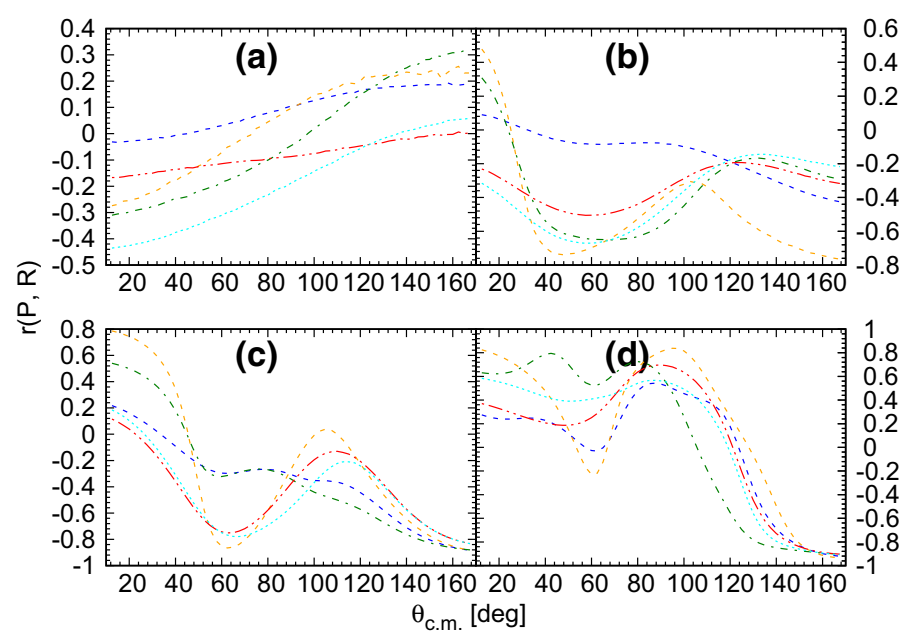

Fig. 2 Same as in Fig. 1, but for the $(P, R)$ pair
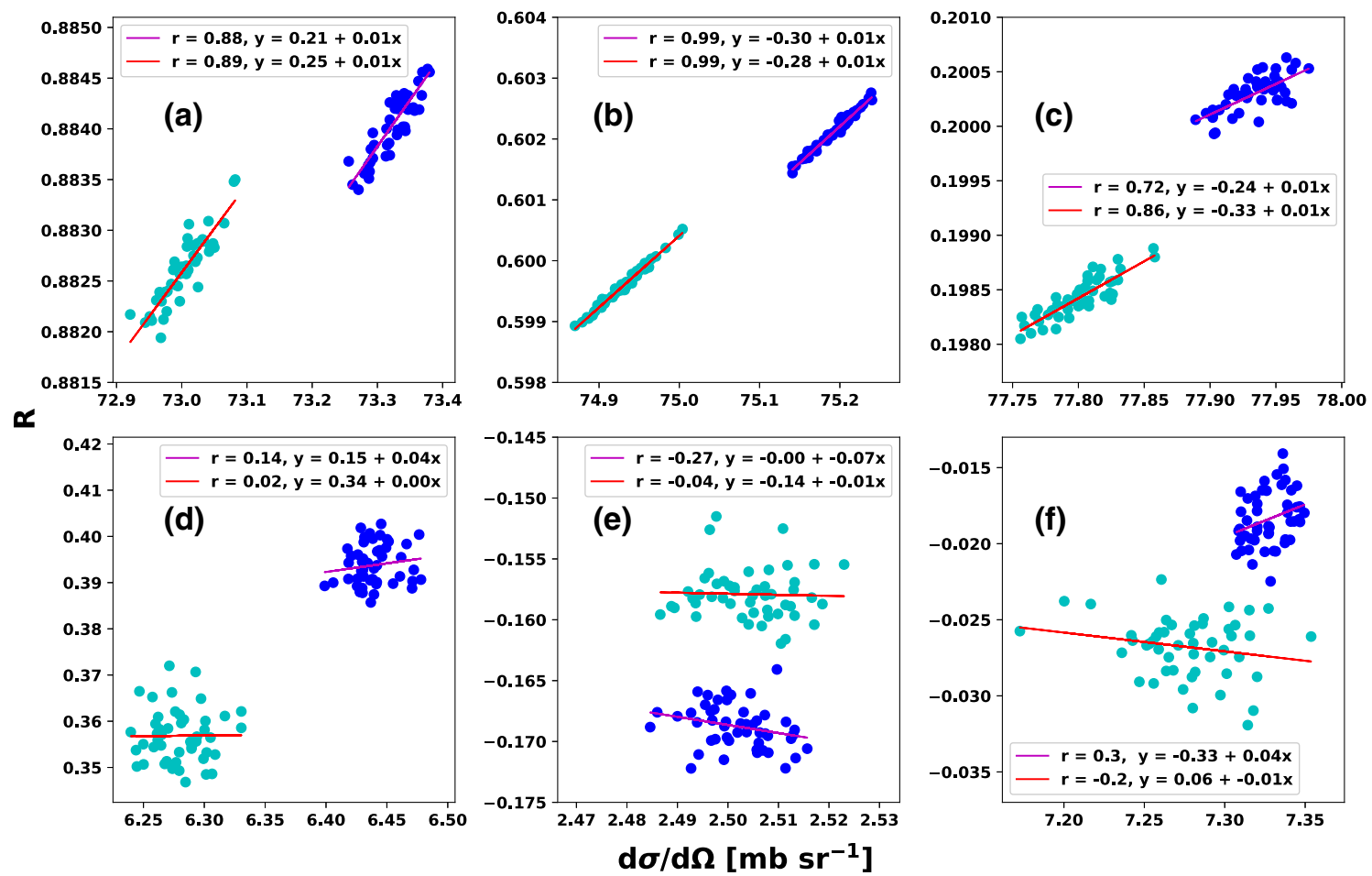

Fig. 3 The scatter plots for the $n p$ differential cross section $d \sigma / d \Omega$ and the depolarization $R$ at the $\theta_{\text {c.m. }}=30^{\circ}$ (left), $\theta_{\text {c.m. }}=90^{\circ}$ (middle) and $\theta_{\text {c.m. }}=150^{\circ}$ (right) scattering angle and at the incident neutron laboratory energy $E_{\text {lab }}=10 \mathrm{MeV}$ (top) and $E_{\text {lab }}=$ $135 \mathrm{MeV}$ (bottom). The cyan and blue circles represent 50 sets of potential parameters of the chiral N4LO $\mathrm{LO}^{+}(\Lambda=450 \mathrm{MeV})$ SMS force and the OPE-Gaussian potential, respectively. The red and magenta lines of best fit correspond to the chiral N4LO ${ }^{+}$ SMS and the OPE-Gaussian models, respectively

OPE-Gaussian potential). However, for the remaining scattering angles, the relation between $P$ and $R$ looks moderate/weak correlation for both potentials, depending on angle.

\section{Conclusions}

We analyzed correlations among the chosen $2 \mathrm{~N}$ observables. Summarizing all above given observations for $2 \mathrm{~N}$ scattering observables we conclude that the angular dependence of the correlation coefficients has a complex 

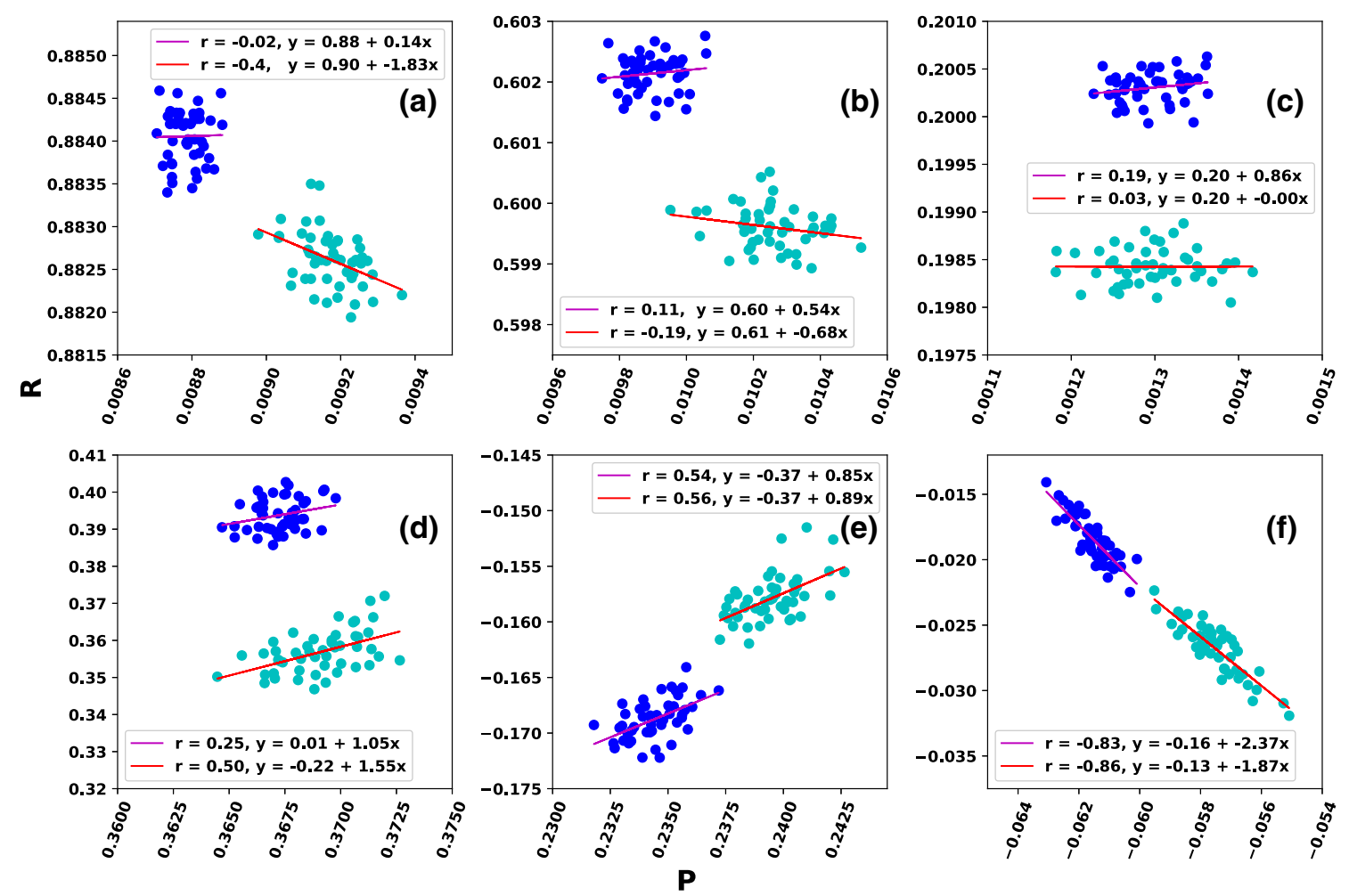

Fig. 4 Same as in Fig. 3, but for the $(P, R)$ pair

behavior depending on the scattering angle and the scattering energy. The differential cross section $d \sigma / d \Omega$ is strongly correlated with the depolarization $R$ at energies up to $E_{\text {lab }}=30 \mathrm{MeV}$, in specific intervals of $\theta_{c . m .}$. The polarisation $P$ is characterized by a weak correlation with $R$, except selected scattering angles at $E_{\text {lab }}=135 \mathrm{MeV}$ where we observe a moderate or even strong relation between them. In the case of the chiral SMS interaction the magnitude of $r$ increases at some scattering angles at increasing orders of chiral expansion. The predictions of the OPE-Gaussian potential are in good agreement with the chiral N4LO ${ }^{+} \mathrm{SMS}$ ones. This is interesting that we have found pairs of $2 \mathrm{~N}$ observables which are strongly correlated or remain uncorrelated independently of the model of the nuclear force and the reaction energy. We believe the reason for this is sensitivity of various observables to different partial waves (different potential parameters) contributing to the scattering amplitude. We hope that the results of our study will be useful for fitting procedures aiming at determining $\mathrm{NN}$ potential parameters, especially in the context of chiral interactions.

Acknowledgements This work is a part of the LENPIC project and was supported by the Polish National Science Center under Grants No. 2016/22/M/ST2/00173. We would like to thank P. Reinert for providing us with the potential subroutine, and Dr. E. Ruiz Arriola and Dr. R. Navarro Pérez for sending us sets of parameters for the OPE-Gaussian potential. The numerical calculations were partially performed on the supercomputer cluster of the JSC, Jülich, Germany.

Open Access This article is licensed under a Creative Commons Attribution 4.0 International License, which permits use, sharing, adaptation, distribution and reproduction in any medium or format, as long as you give appropriate credit to the original author(s) and the source, provide a link to the Creative Commons licence, and indicate if changes were made. The images or other third party material in this article are included in the article's Creative Commons licence, unless indicated otherwise in a credit line to the material. If material is not included in the article's Creative Commons licence and your intended use is not permitted by statutory regulation or exceeds the permitted use, you will need to obtain permission directly from the copyright holder. To view a copy of this licence, visit http://creativecommons.org/licenses/by/4.0/.

\section{References}

1. R. Navarro Pérez et al., Statistical error analysis for phenomenological nucleon-nucleon potentials. Phys. Rev. C 89, 064006 (2014) 
2. P. Reinert et al., Semilocal momentum-space regularized chiral two-nucleon potentials up to fifth order. Eur. Phys. J. A 54, $1-49(2018)$

3. Y. Volkotrub et al., Correlations among observables in two- and three-nucleon systems. Acta Phys. Pol. B 51, 273 (2020)

4. Y. Volkotrub et al., Correlations among 3N observables. Sci. Post Phys. Proc. 3, 055 (2020)

5. Y. Volkotrub et al., Correlations among observables two-nucleon scattering observables. Acta Phys. Pol. B Proc. Suppl. 13, $971(2020)$

6. R. Skibiński et al., Theoretical uncertainties of the elastic nucleon-deuteron scattering observables. Phys. Rev. C 98, 014001 (2018)

7. Y. Volkotrub et al., Uncertainty of three-nucleon continuum observables arising from uncertainties of two-nucleon potential parameters. J. Phys. G Nucl. Part. Phys. 47, 014001 (2020)

8. W. Glöckle, The Quantum Mechanical Few-Body Problem (Springer-Verlag, Berlin Heidelberg, 1983), p. 207

Publisher's Note Springer Nature remains neutral with regard to jurisdictional claims in published maps and institutional affiliations. 